\title{
Resource System with Losses in a Random Environment
}

\author{
Valeriy Naumov ${ }^{1}$ and Konstantin Samouylov $2,3, *$ \\ 1 Service Innovation Research Institute, Annankatu 8 A, 00120 Helsinki, Finland; valeriy.naumov@pfu.fi \\ 2 Applied Informatics and Probability Department, Peoples' Friendship University of Russia \\ (RUDN University), Miklukho-Maklaya St. 6, 117198 Moscow, Russia \\ 3 Institute of Informatics Problems, Federal Research Center "Computer Science and Control" of the Russian \\ Academy of Sciences, Vavilov St. 44-2, 119333 Moscow, Russia \\ * Correspondence: samuylov-ke@rudn.ru
}

check for

updates

Citation: Naumov, V.; Samouylov, K Resource System with Losses in a Random Environment. Mathematics 2021, 9, 2685. https://doi.org/ $10.3390 /$ math 9212685

Academic Editor: Daniel-Ioan Curiac

Received: 14 September 2021

Accepted: 19 October 2021

Published: 22 October 2021

Publisher's Note: MDPI stays neutral with regard to jurisdictional claims in published maps and institutional affiliations.

Copyright: (C) 2021 by the authors Licensee MDPI, Basel, Switzerland. This article is an open access article distributed under the terms and conditions of the Creative Commons Attribution (CC BY) license (https:/ / creativecommons.org/licenses/by/ $4.0 /)$.

\begin{abstract}
The article deals with queueing systems with random resource requirements modeled as bivariate Markov jump processes. One of the process components describes the service system with limited resources. Another component represents a random environment that submits multi-class requests for resources to the service system. If the resource request is lost, then the state of the service system does not change. The change in the state of the environment interacting with the service system depends on whether the resource request has been lost. Thus, unlike in known models, the service system provides feedback to the environment in response to resource requests. By analyzing the properties of the system of integral equations for the stationary distribution of the corresponding random process, we obtain the conditions for the stationary distribution to have a product form. These conditions are expressed in the form of three systems of nonlinear equations. Several special cases are explained in detail.
\end{abstract}

Keywords: product form; loss system; resource requirement; random environment; Marked Markovian Arrival Process

\section{Introduction}

Parameters of queuing systems operating in a random environment can change under the influence of external factors. Such systems are described by two-component random processes, the first component of which represents the external environment and the second describes the service system itself. A Markov chain with a finite number of states is usually considered as a model of the environment. As for the service systems, their models are very diverse: from simple systems described by the birth and death processes $[1,2]$ to more complex processes [3-10]. It is substantially easier to deal with such service systems if the stationary distribution of the underlying process is of product form. A loss system in which the rate of the Poisson arrival process and the service rate both depend on the states of the system and the environment was studied in [11]. The paper provided a condition under which the joint distribution of the environmental state and the set of busy servers has a product form. Product-form conditions for multi-server systems with finite waiting room were analyzed in $[12,13]$. The environmental state space of systems studied in $[12,13]$ is partitioned into two disjoint subsets so that the service process is suspended or resumed depending on which subset of states the environment is in. The Stochastic Automata Network (SAN) formalism provides an efficient technique for studying quantitative characteristics of networks in which each node interacts with only a limited number of other nodes. The product form conditions for SAN in which transition rates in a node also depend on the states of other nodes were obtained in [14-16].

In this article we investigate the interaction between an external environment and a loss system, in which arriving customers may be lost due to lack of resources. Many papers were published since the first research in this area [17]. Numerous references to papers in this area can be found in [18-20]. Systems with random multi-resource requirements are 
widely used in the analysis of modern wireless communication systems [20-23]. In [24] the service process of loss systems with random resource requirements is represented by means of a list of random length consisting of generally distributed random vectors of the quantities of occupied resources. In this article we generalize this model. To specify the considered system, we employ the concept of a "tentative state" [25]. Let all feasible states of the service system form a subset of some set of tentative states. A local transition in the environment does not affect the state of the service system, whereas so-called synchronized transitions correspond to simultaneous state changes of the environment and the loss system. At the time of a synchronized transition, a new state of the environmental process and a tentative state of the loss system are chosen randomly. If the tentative state of the loss system is feasible, then the chosen states are accepted and the transition is finalized. Otherwise, a new state of the environment process is chosen, whereas the state of the loss system remains unchanged. Thus, changes in the random environment at the time of interaction depend on whether the tentative state of the loss system is feasible or not. This provides feedback from the service system to the external environment.

In the next section we discuss stochastic lists of multiple resources, which are commonly used to model queueing systems with random resource requirements. Section 3 introduces the model of a loss system in a random environment with synchronized transitions of several classes. In Section 4 we derive the conditions for the stationary distribution of the underlying process to have a product form. Section 5 details the case of singleclass synchronized transitions, while in Section 6 we specifically address systems without constraints. Section 7 concludes the article.

In what follows boldface lower-case letters denote vectors, and boldface capitals represent matrices. We denote by $\mathbf{u}$ the column vector of ones, and let $\chi(y, E)=1$, if $y \in$ $E$ and $\chi(y, E)=0$ otherwise. Finally, we denote $\delta(i, j)=\chi(i,\{j\})$.

\section{Stochastic Lists of Resources}

Let $\mathcal{C} \subset \mathbb{R}^{M}$ denote a closed Borel subset including the zero vector, which will be referred to as the constraint set. Some examples of constraint sets are $\mathcal{C}_{1}=\left\{\mathbf{x} \in \mathbb{R}^{M} \mid \mathbf{x} \leq \mathbf{c}\right\}$ and $\mathcal{C}_{2}=\left\{\mathbf{x} \in \mathbb{R}^{M} \mid \mathbf{0} \leq \mathbf{x} \leq \mathbf{c}\right\}$, where $\mathbf{c} \in \mathbb{R}^{M}$ is some nonnegative vector. Now, a list of resources of length $n$ is an ordered $n$-tuple $\mathbf{y}=\left(\mathbf{y}_{1}, \mathbf{y}_{2}, \ldots, \mathbf{y}_{n}\right)$ of vectors $\mathbf{y}_{k}=\left(y_{1, k}, y_{2, k}, \ldots, y_{M, k}\right) \in \mathbb{R}^{M}$ such that $\mathbf{y}_{1}+\mathbf{y}_{2}+\ldots+\mathbf{y}_{n} \in \mathcal{C}$ [24]. Note that a list of resources can also be represented by a single vector $\mathbf{y}=\left(y_{1,1}, y_{2,1}, \ldots, y_{M, 1}, \ldots, y_{1, n}, y_{2, n}, \ldots\right.$, $\left.y_{M, n}\right) \in \mathbb{R}^{n M}$ such that $\mathbf{B}_{n} \mathbf{y} \in \mathcal{C}$, where $\mathbf{B}_{n}=\left[B_{n}(i, j)\right]$ is an $M \times n M$ matrix whose elements are

$$
B_{n}(i, j)=\left\{\begin{array}{lc}
1, & j \in\{i, i+M, \ldots, i+(n-1) M\} \\
0, & \text { otherwise. }
\end{array}\right.
$$

Numerous operations can be performed on lists [26]. The most common are lists with the operations of insertion and deletion of an element performed according to the FIFO discipline. For such lists, insertion of element $\mathbf{r} \in \mathbb{R}^{M}$ into a list $\mathbf{y}=\left(\mathbf{y}_{1}, \mathbf{y}_{2}, \ldots, \mathbf{y}_{n}\right)$ results in the list $\mathbf{y}^{\prime}=\left(\mathbf{y}_{1}, \mathbf{y}_{2}, \ldots, \mathbf{y}_{n}, \mathbf{r}\right)$, while deletion from $\mathbf{y}=\left(\mathbf{y}_{1}, \mathbf{y}_{2}, \ldots, \mathbf{y}_{n}\right)$ results in $\mathbf{y}^{\prime \prime}=\left(\mathbf{y}_{2}, \mathbf{y}_{3}, \ldots, \mathbf{y}_{n}\right)$. Insertion of $\mathbf{r}=\left(r_{1}, r_{2}, \ldots, r_{M}\right)$ into a list can be interpreted as allocating amount $r_{k}$ of class $k$ resources, $k=1,2, \ldots, M$, whereas deletion represents releasing corresponding amounts of resources.

In what follows, $\mathscr{Y}_{n}=\mathbb{R}^{n M}$ denotes the set of lists of length $n ; \mathscr{Y}=\cup_{0}^{\infty} \mathscr{Y}_{n}$ is the set of all lists; $S_{n} \subset \mathscr{Y}_{n}$ is the set of lists of length $n$ such that $\mathbf{y}_{1}+\mathbf{y}_{2}+\ldots+\mathbf{y}_{n} \in \mathcal{C} ; \mathscr{B}\left(\boldsymbol{S}_{n}\right)$ denotes the sigma-algebra of Borel subsets of $\mathbb{R}^{n M}$ contained in $\boldsymbol{S}_{n} ; \boldsymbol{S}=\cup_{0}^{L} \boldsymbol{S}_{n}$ is the set of all feasible lists $\mathbf{y}=\left(\mathbf{y}_{1}, \mathbf{y}_{2}, \ldots, \mathbf{y}_{n}\right)$, i.e., of such lists whose length $n$ is not larger than $L$ and for which $\mathbf{y}_{1}+\mathbf{y}_{2}+\ldots+\mathbf{y}_{n} \in \mathcal{C}$; finally, $\mathscr{B}(S)$ denotes the sigma-algebra of subsets of $S$ generated by the sigma-algebras $\mathscr{B}\left(S_{n}\right)$ see Figure 1. 


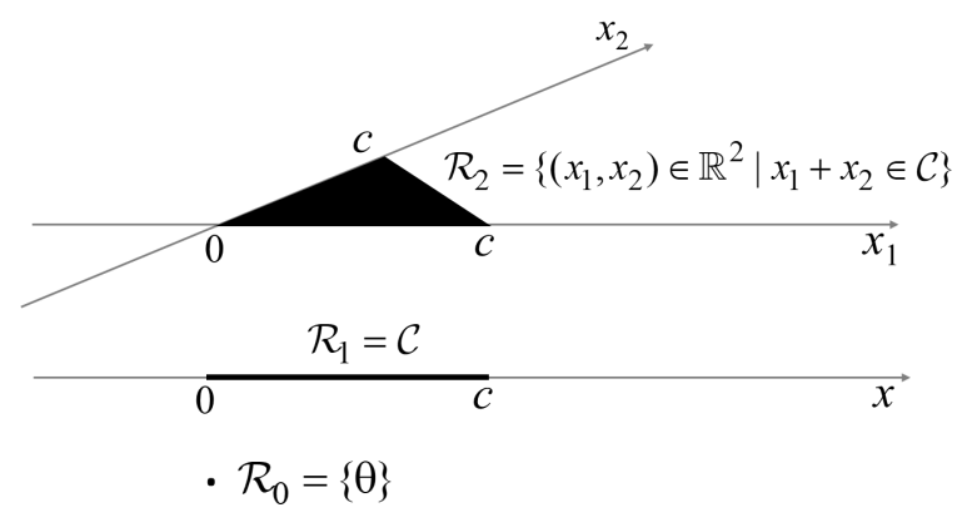

Figure 1. The state space $S=\cup_{0}^{L} S_{n}$ of a stochastic list with $M=1, L=2$ and the constraint set $\mathcal{C}=\{x \in \mathbb{R} \mid 0 \leq x \leq c\}$ is shown in black. Symbol $\theta$ denotes the empty list.

A stochastic process $Y(t)=\left(\zeta_{1}(t), \ldots, \zeta_{n(t)}(t)\right)$ on the state space $S$ is called a stochastic list of resources if each next state of $Y(t)$ is obtained from the previous state through either insertion or deletion of an element [24], and, furthermore, its vector of allocated totals, $\boldsymbol{\sigma}(t)=\zeta_{1}(t)+\ldots+\zeta_{n(t)}(t)$, at all times satisfies the resource constraint $\boldsymbol{\sigma}(t) \in \mathcal{C}$. Queueing systems with random resource requirements are described by processes of the form $Z(t)=(X(t), Y(t))$, where $X(t)$ represents the state of the queueing system at time $t$, and $Y(t)$ is a stochastic list of resources allocated at time $t$. At some times, operations are performed on the list $Y(t)$. Before an operation is finalized, its expected result called a tentative state is established. If the tentative state is such that the resource constraint $\boldsymbol{\sigma}(t) \in \mathcal{C}$ does not hold, then the operation is canceled and the list does not change. The state of the queueing system, $X(t)$, changes at the time of the operation depending on whether or not the operation is finalized.

Various generalizations of the described model are possible. For instance, besides insertion and deletion, an operation of replacing a list element with another one can be introduced. Moreover, list elements of various classes can be considered and constraints can be placed upon both the number of elements of each class and the amount of resources they occupy. Therefore, in the sections that follow we consider a more general model and assume that $Y(t)$, which describes resource allocation in the system, is some Markov jump process on the state space $S \subset \mathscr{Y}$. The set of tentative states $\mathscr{Y}$ is assumed closed with respect to supported operations but can include states that do not satisfy the constraints. Conversely, the set of all feasible states, $S$, is formed by all the elements of $\mathscr{Y}$ satisfying the constraints.

\section{Loss Systems in a Random Environment with Feedback}

Let $\langle\Omega, \mathscr{F}, \mathrm{P}\rangle$ represent a probability space, and $(\mathcal{Z}, \mathscr{G})$ be a measurable space. Consider a jump process $Z(t)$ on $\langle\Omega, \mathscr{F}, \mathrm{P}\rangle$ taking values in $(\mathcal{Z}, \mathscr{G})$ and having right-continuous trajectories, and assume that for any given $T$, there almost surely exist in $(0, T]$ a finite number of points such that in the intervals between them $Z(t)$ is constant. Such a process can be specified by a sequence $Z_{n}, t_{n}, n=0,1, \ldots$, where $Z_{n}=Z\left(t_{n}\right), t_{0}=0$, and $t_{n}, n \geq 1$, are jump times. The difference $\tau_{n}=t_{n+1}-t_{n}$ corresponds to a sojourn time of $Z(t)$ in state $Z_{n}$. Sojourn times in state $z$ are exponentially distributed with parameter $\eta(z)$, and upon leaving state $z$ the process moves into a set of states $G \in \mathscr{G}$ with probability $P(z, G)$. Furthermore, for a homogeneous jump process we have

$P\left\{Z_{r+1} \in G, \tau_{r}<x \mid \tau_{0}=x_{0}, \ldots, \tau_{r-1}=x_{r-1}, Z_{0}=z_{0}, \ldots, Z_{r-1}=z_{r-1}, Z_{r}=z_{r}\right\}=\left(1-e^{-x \eta\left(z_{r}\right)}\right) P\left(z_{r}, G\right)$ 
for all states $z_{0}, z_{1}, \ldots, z_{r} \in \mathscr{Z}$, a subset $G \in \mathscr{G}$ and some scalars $x_{0}, x_{1}, \ldots, x_{r-1}>0$ [27]. Here, $\eta(z)$ is a positive function, and $P(z, G)$ is a stochastic kernel. The stationary distribution, $p(G), G \in \mathscr{G}$, of $Z(t)$ solves the system of equilibrium equations

$$
\int_{G} \eta(z) p(d z)=\int_{\mathcal{Z}} \eta(z) P(z, G) p(d z), G \in \mathscr{G} .
$$

Now, let $\mathscr{X}$ represent a non-empty finite set, $(\mathscr{Y}, \mathscr{B})$ a measurable space, $S \in \mathscr{B}$ a non-empty subset of $\mathscr{Y}, \mathscr{B}(S)=\{E \in \mathscr{B} \mid E \subset S\}$, and $\bar{S}=\mathscr{Y} \backslash S$. Consider a loss system in a random environment governed by a homogeneous Markov jump process $Z(t)=(X(t), Y(t))$ on state space $\mathscr{Z}=\mathscr{X} \times S$ with the following properties: (1) the departure rate $\eta(i, y)$ of $Z(t)$ from any state $z=(i, y)$ depends on the value of its first component only, i.e., $\eta(i, y)=v(i)$, and (2) the transition probabilities $P_{i j}(y, E)=P((i, y),\{j\} \times E)$ of $Z(t)$ at a jump time are given by

$$
\begin{gathered}
P_{i j}(y, E)=\sum_{k=1}^{K} a_{k}(i)\left(p_{k}(i, j) G_{k}(y, E)+\bar{p}_{k}(i, j) G_{k}(y, \bar{S}) \chi(y, E)\right) \\
+a_{0}(i) p_{0}(i, j) \chi(y, E), i, j \in \mathscr{X}, y \in S, E \in \mathscr{B}(\boldsymbol{S})
\end{gathered}
$$

where $a_{0}(i), a_{1}(i), \ldots, a_{K}(i)$ is some probability distribution, $\sum_{k=0}^{K} a_{k}(i)=1, i \in \mathscr{X}, \mathbf{P}_{k}=$ $\left[p_{k}(i, j)\right]_{i, j \in \mathscr{X}}, 0 \leq k \leq K$, and $\overline{\mathbf{P}}_{k}=\left[\bar{p}_{k}(i, j)\right]_{i, j \in \mathscr{X}}, 1 \leq k \leq K$, are stochastic matrices, and $G_{k}(y, E), y \in \mathscr{Y}, E \in \mathscr{B}, 1 \leq k \leq K$, are transition kernels.

The stochastic process $Z(t)=(X(t), Y(t))$ has state transitions of $K+1$ classes. A jump from state $(i, y)$ results either in a local transition of the environmental state, with probability $a_{0}(i)$, or in a synchronized transition of class $k, 1 \leq k \leq K$, with probability $a_{k}(i)$. Local transitions of the environmental state are specified by the transition probability matrix $\mathbf{P}_{0}$. Upon a synchronized transition of class $k$, a state of the environment and a tentative state of the service process are chosen according to transition probability matrix $\mathbf{P}_{k}$ and probability kernel $G_{k}(y, E)$ respectively. If the tentative state of the service system belongs to $S$, these chosen states are accepted. If, on the contrary, the tentative state does not belong to $S$, then the environmental state change is governed by the transition probability matrix $\overline{\mathbf{P}}_{k}$, while the state of the service system remains unchanged.

The above model is particularly suitable for describing queueing systems with random resource requirements. While the process $X(t)$ represents the behavior of the external environment, the process $Y(t)$ models resource allocation to customers. Upon a synchronized transition, the service system receives a request for allocating or releasing resources needed for serving customers. The transition kernels $G_{k}(y, E)$ are used to determine new states of the service system. If the new state belongs to $S$, then the request is granted and the transition in the service system takes place. Otherwise, the request is rejected and the state of the service system remains unchanged. State changes of the environment are governed by either $\mathbf{P}_{k}$ or $\overline{\mathbf{P}}_{k}$ providing feedback from the service system to the external environment.

In what follows, we will need matrices $\mathbf{A}_{k}=\left[A_{k}(i, j)\right]_{i, j \in \mathscr{X}}$ and $\overline{\mathbf{A}}_{k}=\left[\bar{A}_{k}(i, j)\right]_{i, j \in \mathscr{X}}$ whose elements are given by

$$
\begin{gathered}
A_{0}(i, j)=v(i) a_{0}(i) p_{0}(i, j)-v(i) \delta(i, j), i, j \in \mathscr{X}, \\
A_{k}(i, j)=v(i) a_{k}(i) p_{k}(i, j), \bar{A}_{k}=v(i) a_{k}(i) \bar{p}_{k}(i, j), i, j \in \mathscr{X}, 1 \leq k \leq K .
\end{gathered}
$$

\section{Product Form of the Stationary Distribution}

Let the stationary probabilities $p(\{i\} \times E), i \in \mathscr{X}, E \in \mathscr{B}(S)$, of $Z(t)$ form vectors $\mathbf{p}(E)=(p(\{i\} \times E))_{i \in \mathscr{X}}$, and rewrite the equilibrium Equation (1) as

$$
\mathbf{p}(E) \mathbf{A}_{0}+\sum_{k=1}^{K}\left(\int_{S} G_{k}(y, E) \mathbf{p}(d y) \mathbf{A}_{k}+\int_{E} G_{k}(y, \bar{S}) \mathbf{p}(d y) \overline{\mathbf{A}}_{k}\right)=0, E \in \mathscr{B}(\boldsymbol{S}) .
$$


The following Theorem 1, which we prove in Appendix A, provides the necessary and sufficient conditions for the solution of (3) to be of product form.

Theorem 1. The stationary distribution of the process $Z(t)=(X(t), Y(t))$ is of a product form $\mathbf{p}(E)=Q(E) \mathbf{q}, E \in \mathscr{B}(S)$, where $\mathbf{q}=\left(q_{i}\right)_{i \in \mathscr{X}}$ and $Q(S)=1$, if and only if the probability measure $Q(E), E \in \mathscr{B}(S)$, and the stochastic vector $\mathbf{q}$ satisfy

$$
\begin{gathered}
\mathbf{q}\left(\mathbf{A}_{0}+\sum_{k=1}^{K}\left(G_{k}(\boldsymbol{S}) \mathbf{A}_{k}+\bar{G}_{k}(\boldsymbol{S}) \overline{\mathbf{A}}_{k}\right)\right)=\mathbf{0} \\
\lambda_{0} Q(E)+\sum_{k=1}^{K} \lambda_{k}\left(G_{k}(E)+\bar{G}_{k}(E)\right)=0, E \in \mathscr{B}(\boldsymbol{S}), \\
\sum_{k=1}^{K}\left(G_{k}(E)-G_{k}(\boldsymbol{S}) Q(E)\right)\left(\mathbf{q} \mathbf{A}_{k}-\lambda_{k} \mathbf{q}\right)+\sum_{k=1}^{K}\left(\bar{G}_{k}(E)-\bar{G}_{k}(\boldsymbol{S}) Q(E)\right)\left(\mathbf{q} \overline{\mathbf{A}}_{k}-\lambda_{k} \mathbf{q}\right)=\mathbf{0}, \\
E \in \mathscr{B}(\boldsymbol{S}),
\end{gathered}
$$

where

$$
\begin{aligned}
& \lambda_{i}=\mathbf{q A}_{i} \mathbf{u}, 0 \leq i \leq K ; G_{k}(E)=\int_{S} G_{k}(y, E) Q(d y), \bar{G}_{k}(E)=\int_{E} G_{k}(y, \bar{S}) Q(d y), \\
& 1 \leq k \leq K \text {. }
\end{aligned}
$$

Condition (4) means that if the stationary distribution of $Z(t)=(X(t), Y(t))$ has the form $\mathbf{p}(E)=Q(E) \mathbf{q}$, then the stationary probabilities $\mathbf{q}$ of the environmental states are the same as the stationary distribution of a Markov chain with the generator

$$
\mathbf{A}=\mathbf{A}_{0}+\sum_{k=1}^{K}\left(G_{k}(\boldsymbol{S}) \mathbf{A}_{k}+\bar{G}_{k}(\boldsymbol{S}) \overline{\mathbf{A}}_{k}\right)
$$

Here, $G_{k}(S)$ and $\bar{G}_{k}(S)$ are the probabilities that the tentative state chosen at a time of synchronized transition of class $k$ will be, respectively, accepted or rejected. According to (5), the stationary distribution $Q(E)$ of the service system's states is the same as if it had Poisson arrivals of synchronized transition epochs with rates $\lambda_{k}, 1 \leq k \leq K$.

\section{The Case of Single-Class Synchronized Transitions}

Assume now that the environment and the loss system interact with each other only via synchronized transitions forming a single class. Then, conditions (4)-(6) of Theorem 1 take on the form

$$
\begin{gathered}
\mathbf{q}\left(\mathbf{A}_{0}+\int_{S} G_{1}(y, S) Q(d y) \mathbf{A}_{1}+\int_{S} G_{k}(y, \overline{\boldsymbol{S}}) Q(d y) \overline{\mathbf{A}}_{1}\right)=\mathbf{0} \\
\lambda_{0} Q(E)+\lambda_{1}\left(\int_{S} G_{1}(y, E) Q(d y)+\int_{E} G_{1}(y, \overline{\boldsymbol{S}}) Q(d y)\right)=0, E \in \mathscr{B}(\boldsymbol{S}) \\
\left(\int_{S} G_{1}(y, E) Q(d y)-Q(E) \int_{S} G_{k}(y, S) Q(d y)\right)\left(\mathbf{q} \mathbf{A}_{1}-\lambda_{1} \mathbf{q}\right) \\
+\left(\int_{E} G_{1}(y, \overline{\boldsymbol{S}}) Q(d y)-Q(E) \int_{S} G_{k}(y, \overline{\boldsymbol{S}}) Q(d y)\right)\left(\mathbf{q} \overline{\mathbf{A}}_{1}-\lambda_{1} \mathbf{q}\right)=0, E \in \mathscr{B}(\boldsymbol{S}) .
\end{gathered}
$$

The following theorem, which specifies the product-form conditions for the system with single-class synchronized transitions, is proved in Appendix B. 
Theorem 2. Let the number of classes $K$ equal one, and let a stochastic vector $\mathbf{q}$ and a probability measure $Q(E), E \in \mathscr{B}(S)$, solve Equations (9) and (10). Then, for the stationary distribution of the process $Z(t)$ to be of product form, it is necessary and sufficient that at least one of the following conditions hold:

1. $\quad \mathbf{q A}_{1}=\mathbf{q} \overline{\mathbf{A}}_{1}=\lambda_{1} \mathbf{q} ;$

2. $\quad \mathbf{q A}_{1}=\lambda_{1} \mathbf{q}$ and $\int_{E} G_{1}(y, \overline{\boldsymbol{S}}) Q(d y)=Q(E) \int_{S} G_{1}(y, \overline{\boldsymbol{S}}) Q(d y)$ for all $E \in \mathscr{B}(\boldsymbol{S})$;

3. $\mathbf{q} \overline{\mathbf{A}}_{1}=\lambda_{1} \mathbf{q}$ and $\int_{S} G_{1}(y, E) Q(d y)=Q(E) \int_{S} G_{1}(y, S) Q(d y)$ for all $E \in \mathscr{B}(S)$;

4. $\int_{S} G_{1}(y, E) Q(d y)=Q(E) \int_{S} G_{1}(y, S) Q(d y)$ and $\int_{E} G_{1}(y, \bar{S}) Q(d y)=Q(E) \int_{S} G_{1}(y, \bar{S})$ $Q(d y)$ for all $E \in \mathscr{B}(S)$;

5. There exists a constant $\psi \neq 0$ such that $\sum_{i \in \mathscr{X}} q(i) \bar{A}_{1}(i, j)-\lambda_{1} q(j)=\psi\left(\sum_{i \in \mathscr{X}} q(i) A_{1}(i, j)-\right.$ $\left.\lambda_{1} q(j)\right)$ for all $j \in \mathscr{X}$ with $\sum_{i \in \mathscr{X}} q(i) \bar{A}_{1}(i, j) \neq \lambda_{1} q(j)$, and $Q(E) \int_{S} G_{1}(y, S) Q(d y)-\int_{S} G_{1}$ $(y, E) Q(d y)=\psi\left(\int_{E} G_{1}(y, \overline{\boldsymbol{S}}) Q(d y)-Q(E) \int_{S} G_{1}(y, \bar{S}) Q(d y)\right)$ for all $E \in \mathscr{B}(S)$ with $\int_{S} G_{1}$ $(y, E) Q(d y) \neq Q(E) \int_{S} G_{1}(y, S) Q(d y)$.

\section{Unconstrained Systems}

If $S=\mathscr{Y}$, then we refer to the model under study as unconstrained system. The transition probabilities of the underlying process $Z(t)$ can then be obtained from (2) by letting $G_{k}(y, \overline{\mathbf{S}})=0$, which yields

$$
\begin{gathered}
P_{i j}(y, E)=a_{0}(i) p_{0}(i, j) \chi(y, E)+\sum_{k=1}^{K} a_{k}(i) p_{k}(i, j) G_{k}(y, E), i, j \in \mathscr{X}, \\
y \in \mathscr{Y}, E \in \mathscr{B} .
\end{gathered}
$$

For an unconstrained system, the following transition probabilities do not depend on $y$ :

$$
P_{i j}(y, \mathscr{Y})=\sum_{k=0}^{K} a_{k}(i) p_{k}(i, j), i, j \in \mathscr{X}, y \in \mathscr{Y}, E \in \mathscr{B} .
$$

Moreover, the departure rate $v(i)$ of $Z(t)$ from any state $z=(i, y)$ depends only on the state of $X(t)$. Hence, the process $Z(t)=(X(t), Y(t))$, which models an unconstrained system, is lumpable and its first component, $X(t)$, is a Markov chain with a generator

$$
\mathbf{A}=\sum_{k=0}^{K} \mathbf{A}_{k}
$$

Furthermore, the times of synchronized transitions form a Marked Markovian Arrival Process (MMAP) characterized by the matrices $\mathbf{A}_{k}, k=0,1, \ldots, K$ [28-30].

In the case of an unconstraint system, we have $S=\mathscr{Y}, \bar{S}=\varnothing, G_{k}(S)=1$ and $\bar{G}_{k}(E)=0$ for all $E \in \mathscr{B}$. Hence, conditions (4)-(6) of Theorem 1 assume the form

$$
\begin{gathered}
\mathbf{q A}=0 ; \lambda_{0} Q(E)+\sum_{k=1}^{K} \lambda_{k} \int_{\mathscr{Y}} G_{k}(y, E) Q(d y)=0, E \in \mathscr{B} ; \\
\sum_{k=1}^{K}\left(\int_{\mathscr{Y}} G_{k}(y, E) Q(d y)-Q(E)\right)\left(\mathbf{q} \mathbf{A}_{k}-\lambda_{k} \mathbf{q}\right)=0, E \in \mathscr{B} ;
\end{gathered}
$$

Since the times of transitions of each class form a Markovian Arrival Process, the relationship $\mathbf{q A}_{k}=\lambda_{k} \mathbf{q}$ holds only if the stream of epochs of class $k$ synchronized transitions in steady state is Poisson with rate $\lambda_{k}[30,31]$. 


\section{Conclusions}

In this article, we have studied a loss system in a random environment such that upon receiving a request for resources from the environment, the service process informs the environment whether the request has been accepted. Upon a request arrival, a tentative state of the service system is randomly chosen from a set that contains its state space but may also include unfeasible states. If the chosen state is feasible, then the state change is accepted and the transition occurs. Otherwise, the state of the service system does not change. The way the environmental state changes depend on whether or not the state of the service system has changed. We have derived the necessary and sufficient conditions for the stationary probability distribution of the underlying Markov jump process to be of product form. In this case, the stationary distribution of the environmental state will be the same as if the probability of accepting feasible state depended only on the type of synchronized transition, and not on the state of the service process. Moreover, the stationary state distribution of the service system is the same as if synchronized transition epochs followed Poisson processes with rates depending of the transition type.

At first glance, the paper is purely theoretical. However, our work on various aspects of the theory of resource queueing systems has reveals a large area of applications thereof, namely for performance analysis of 5th generation wireless networks. Due to the limited space of the paper, we have not discussed examples of these highly interesting applications here. The reader is referred to articles [21-23], recently published in the IEEE series of journals, where one can find descriptions of system models, formulations of mathematical problems, methods for their analysis and numerical estimates of the metrics under study.

Author Contributions: Conceptualization, V.N. and K.S.; Formal analysis, V.N.; Investigation, V.N. (Sections 2-4) and K.S. (Sections 1 and 5-7); Methodology, V.N. and K.S. All authors have read and agreed to the published version of the manuscript.

Funding: This paper has been supported by the RUDN University Strategic Academic Leadership Program (recipient K.S., Sections 1, 5 and 6). The reported study has been funded by RFBR, project 19-07-00933 (recipient K.S., Section 7).

Conflicts of Interest: The authors declare no conflict of interest.

\section{Appendix A}

Proof of Theorem 1. Assume that $\mathbf{p}(E)=Q(E) \mathbf{q}$, where $Q(S)=1$, for any $E \in \mathscr{B}(S)$. Then, it follows from (2) and (7) that the quantity (8) has the properties

$$
\bar{g}_{k}=1-g_{k}, \lambda_{k}=\mathbf{q} \overline{\mathbf{A}}_{k} \mathbf{u}, 1 \leq k \leq K, \lambda_{0}=-\sum_{k=1}^{K} \lambda_{k}
$$

Assuming $\mathbf{p}(E)=Q(E) \mathbf{q}, E \in \mathscr{B}(S)$, we can rewrite the equilibrium Equation (3) as

$$
Q(E) \mathbf{q} \mathbf{A}_{0}+\sum_{k=1}^{K}\left(G_{k}(E) \mathbf{q} \mathbf{A}_{k}+\bar{G}_{k}(E) \mathbf{q} \overline{\mathbf{A}}_{k}\right)=\mathbf{0}
$$

By letting $E=S$ in (A2) we obtain (4). In addition, by multiplying both sides of (A2) on the right by vector $\mathbf{u}$ we obtain (5). The conditions (4) and (5) are thus necessary for a product-form stationary distribution.

Let us now prove the necessity of (6). By letting $E=S$ in (5) we have

$$
\lambda_{0}+\sum_{k=1}^{K}\left(\lambda_{k} g_{k}+\lambda_{k} \bar{g}_{k}\right)=0
$$


Now, we subtract from the left-hand and right-hand sides of (A2) the respective sides of (4) multiplied by $Q(E)$, which yields

$$
\sum_{k=1}^{K}\left(G_{k}(E)-g_{k} Q(E)\right) \mathbf{q} \mathbf{A}_{k}+\sum_{k=1}^{K}\left(\bar{G}_{k}(E)-\bar{g}_{k} Q(E)\right) \mathbf{q} \overline{\mathbf{A}}_{k}=\mathbf{0} .
$$

We then right multiply by $\mathbf{q}$ the left-hand and right-hand sides of (5) and subtract from the resulting relationships the respective sides of (A3) multiplied by $Q(E) \mathbf{q}$. This yields

$$
\sum_{k=1}^{K} \lambda_{k}\left(G_{k}(E)-g_{k} Q(E)\right) \mathbf{q}+\sum_{k=1}^{K} \lambda_{k}\left(\bar{G}_{k}(E)-\bar{g}_{k} Q(E)\right) \mathbf{q}=\mathbf{0} .
$$

Finally, by subtracting from the left-hand and right-hand sides of (A3) the respective sides of (A6) we obtain (6). The condition (6) is thus necessary for the stationary distribution to be of product form.

We now prove that (4)-(6) are sufficient for (A2) to hold. By removing parentheses in (6) we have

$$
\begin{gathered}
\sum_{k=1}^{K} G_{k}(E) \mathbf{q} \mathbf{A}_{k}-\sum_{k=1}^{K} g_{k} Q(E) \mathbf{q} \mathbf{A}_{k}-\sum_{k=1}^{K} \lambda_{k} G_{k}(E) \mathbf{q}+\sum_{k=1}^{K} g_{k} \lambda_{k} Q(E) \mathbf{q} \\
+\sum_{k=1}^{K} \bar{G}_{k}(E) \mathbf{q} \overline{\mathbf{A}}_{k}-\sum_{k=1}^{K} \bar{g}_{k} Q(E) \mathbf{q} \overline{\mathbf{A}}_{k}-\sum_{k=1}^{K} \lambda_{k} \bar{G}_{k}(E) \mathbf{q}+\sum_{k=1}^{K} \bar{g}_{k} \lambda_{k} Q(E) \mathbf{q}=\mathbf{0} .
\end{gathered}
$$

By using (4) and (8), this yields (A2), which proves the theorem.

\section{Appendix B}

Proof of Theorem 2. According to Theorem 1, for a product-form stationary distribution $\mathbf{p}(E)=Q(E) \mathbf{q}, E \in \mathscr{B}(S)$, it is required that for any $j \in \mathscr{X}$ and $E \in \mathscr{B}(S)$ the following holds:

$$
h(j) H(E)+\bar{h}(j) \bar{H}(E)=0,
$$

where

$$
\begin{aligned}
h(j)= & \sum_{i \in \mathscr{X}} q(i) A_{1}(i, j)-\lambda_{1} q(j), \bar{h}(j)=\sum_{i \in \mathscr{X}} q(i) \bar{A}_{1}(i, j)-\lambda_{1} q(j), \\
& H(E)=\int_{S} G_{1}(y, E) Q(d y)-Q(E) \int_{S} G_{1}(y, S) Q(d y), \\
& \bar{H}(E)=\int_{E} G_{1}(y, \overline{\boldsymbol{S}}) Q(d y)-Q(E) \int_{S} G_{1}(y, \overline{\boldsymbol{S}}) Q(d y) .
\end{aligned}
$$

The sufficiency of each of the five conditions for a product-form stationary distribution $\mathbf{p}(E)$ is obvious. Let us prove that at least one of them is necessary for the stationary distribution to be of product form.

Suppose that the stationary distribution $\mathbf{p}(E)$ is of product form. From (A7), it follows that if one of the vectors $\mathbf{h}, \overline{\mathbf{h}}$, is a zero vector or one of the functions $H(E), \bar{H}(E)$, is identically zero, then there is another zero vector or function in the group $\mathbf{h}, \overline{\mathbf{h}}, H(E), \bar{H}(E)$, such that one of the conditions (1)-(4) of Theorem 2 is fulfilled.

It remains to consider the case when all vectors $\mathbf{h}, \overline{\mathbf{h}}$, and functions $H(E), \bar{H}(E)$, are non-zero. Let $\mathscr{V}$ be a set of $j \in \mathscr{X}$ such that $h(j) \neq 0$, and let $\mathscr{W}$ be a set of $E \in \mathscr{B}(S)$ such that $\bar{H}(E) \neq 0$. Then, for all $j \in \mathscr{V}$ and $E \in \mathscr{W}$,

$$
\frac{\bar{h}(j)}{h(j)}=-\frac{H(E)}{\bar{H}(E)} .
$$


The left-hand side of (A8) does not depend on $E$, while the right-hand side does not depend on $j$, therefore, for all $j \in \mathscr{V}$ and $E \in \mathscr{W}$, both sides of (A8) are equal to some constant $\psi$, and hence

$$
\bar{h}(j)=\psi h(j), j \in \mathscr{V}, H(E)=-\psi \bar{H}(E), E \in \mathscr{W} .
$$

Suppose now that $\bar{h}\left(j^{*}\right)=0$ for some $j^{*} \in \mathscr{V}$. Then it follows from (A7) that $H(E)=0$ for all $E \in \mathscr{B}(S)$, which contradicts the assumption that the function $H(E)$ is non-zero. Therefore, $\bar{h}(j) \neq 0$ for all $j \in \mathscr{V}$ and thus $\psi \neq 0$, which proves the theorem.

\section{References}

1. Zolotarev, V.M. Distribution of queue length and number of operating lines in a system of Erlang type with random breakage and restoration of lines. Sel. Transl. Math. Stat. Probab. 1966, 6, 89-99.

2. Yechiali, U. A queueing-type birth-and-death process defied on a continuous-time Markov chain. Oper. Res. 1973, 21, 604-609. [CrossRef]

3. Cogburn, R. Markov Chains in Random Environments: The Case of Markovian Environments. Ann. Probab. 1980, 8, 908-916. [CrossRef]

4. Chistyakov, A.V. Limit theorems for Markov processes in a rapidly changing random environment. Math. USSR-Sb. 1984, 49, 239-254. [CrossRef]

5. Fakinos, D. Insensitivity of generalized semi-Markov processes evolving in a random environment. J. Oper. Res. Soc. 1991, 42, 1111-1115. [CrossRef]

6. Yamazaki, G.; Miyazawa, M. Decomposability in queues with background states. Queueing Syst. 1995, 20, 453-469. [CrossRef]

7. Tkachenko, A.V. Multichannel Queueing System in a Random Environment. Mosc. Univ. Math. Bull. 2014, 69, 37-40. [CrossRef]

8. Kim, C.S.; Dudin, A.; Klimenok, V.; Khramova, V. Erlang loss queueing system with batch arrivals operating in a random environment. Comput. Oper. Res. 2009, 36, 674-697. [CrossRef]

9. Naumov, V.A.; Gaidamaka, Y.V.; Samouylov, K.E. On Two Interacting Markovian Queueing Systems. Mathematics 2019, 7, 799. [CrossRef]

10. Lovasa, A.; Rásonyi, M. Markov chains in random environment with applications in queuing theory and machine learning. Stoch. Process. Appl. 2021, 137, 294-326. [CrossRef]

11. Falin, G. A heterogeneous blocking system in a random environment. J. Appl. Probab. 1996, 33, 211-216. [CrossRef]

12. Krenzler, R.; Daduna, H. Loss systems in a random environment: Steady state analysis. Queueing Syst. 2015, 80, 127-153. [CrossRef]

13. Krenzler, R. Queueing Systems in a Random Environment. Ph.D. Thesis, University of Hamburg, Hamburg, Germany, 2016.

14. Plateau, B. On the Stochastic Structure of Parallelism and Synchronization Models for Distributed Algorithms. In Proceedings of the ACM SIGMETRICS Conference on Measurement and Modeling of Computer Systems, Austin, TX, USA, August 1985; Association for Computing Machinery: New York, NY, USA, 1985; pp. 147-154. [CrossRef]

15. Plateau, B.; Stewart, W.J. Stochastic Automata Networks. In Computational Probability; Grassmann, W.K., Ed.; Springer: Boston, MA, USA, 2000; pp. 113-151.

16. Fourneau, J.M.; Plateau, B.; Stewart, W.J. Product Form for Stochastic Automata Networks. In ValueTools'07; Glynn, P., Tuffin, B., Eds.; ICST: Brussels, Belgium, 2007; pp. 1-10.

17. Omahen, K.J. Analytic Models of Multiple Resource Systems. Ph.D. Thesis, University of Chicago, Chicago, IL, USA, 1973.

18. Kaufman, J. Blocking in a Shared Resource Environment. IEEE Trans. Commun. 1981, 29, 1474-1481. [CrossRef]

19. Choudhury, G.L.; Leung, K.K.; Whitt, W. An Algorithm to Compute Blocking Probabilities in Multi-Rate Multi-Class MultiResource Loss Models. Adv. Appl. Probab. 1995, 27, 1104-1143. [CrossRef]

20. Gorbunova, A.V.; Naumov, V.A.; Gaidamaka, Y.V.; Samouylov, K.E. Resource queuing systems as models of wireless communication systems. Inform. Appl. 2018, 12, 48-55. [CrossRef]

21. Petrov, V.; Solomitckii, D.; Samuylov, A.; Lema, M.A.; Gapeyenko, M.; Moltchanov, D. Dynamic Multi-Connectivity Performance in Ultra-Dense Urban mmWave Deployments. IEEE J. Sel. Areas Commun. 2017, 35, 2038-2055. [CrossRef]

22. Begishev, V.; Sopin, E.; Moltchanov, D.; Kovalchukov, R.; Samuylov, A.; Andreev, S. Joint Use of Guard Capacity and Multiconnectivity for Improved Session Continuity in Millimeter-Wave 5G NR Systems. IEEE Trans. Veh. Technol. 2021, 70, $2657-2672$. [CrossRef]

23. Begishev, V.; Sopin, E.; Moltchanov, D.; Pirmagomedov, R.; Samuylov, A.; Andreev, S. Performance Analysis of Multi-Band Microwave and Millimeter-Wave Operation in 5G NR Systems. IEEE Trans. Wirel. Commun. 2021, 20, 3475-3490. [CrossRef]

24. Samouylov, K.; Naumov, V. Stochastic Lists of Multiple Resources. In Proceedings of the 5th International Conference on Stochastic Methods (ICSM-2020), Moscow, Russia, 23-27 November 2020; Springer: New York, NY, USA, 2021. [CrossRef]

25. Bremaud, P. Markov Chains: Gibbs Fields, Monte Carlo Simulation and Queues; Springer: New York, NY, USA, 2020. [CrossRef]

26. Knuth, D. The Art of Computer Programming, Volume 1: Fundamental Algorithms, 3rd ed.; Addison-Wesley: Reading, MA, USA, 1997.

27. Korolyuk, V.S.; Korolyuk, V.V. Stochastic Models of Systems; Springer: New York, NY, USA, 1999. [CrossRef] 
28. Naumov, V.A. Markovian Modeling of Arrival Processes. In Queuing Systems and Informatics; UDN: Moscow, Russia, 1987; pp. 67-73.

29. He, Q.M. Queues with marked customers. Adv. Appl. Probab. 1996, 28, 567-587. [CrossRef]

30. Basharin, G.P.; Naumov, V.A.; Samouylov, K.E. On Markovian modelling of arrival processes. Stat. Pap. 2018, 59, 1533-1540. [CrossRef]

31. Bean, N.G.; Green, D.A. When is a MAP poisson? Math. Comput. Model. 2000, 31, 31-46. [CrossRef] 